Revista Destaques Acadêmicos, Lajeado, v. 10, n. 4, 2018. ISSN 2176-3070

DOI: http://dx.doi.org/10.22410/issn.2176-3070.v10i4a2018.2029

http://www.univates.br/revistas

\title{
PROJETO ACÚSTICO DE UMA SALA DE CONCERTOS/RECITAIS
}

\author{
Adriano Inéia ${ }^{1}$, Angélica Cristine Ghiggi ${ }^{2}$, Rodrigo Spinelli ${ }^{3}$
}

Resumo: A proposta deste estudo é avaliar e propor um projeto acústico eficiente para uma sala de concerto/recital. As soluções foram adotadas conforme os materiais fornecidos pelo comércio especializado da área. O plano de fundo do estudo é melhorar o condicionamento e desempenho acústico, a fim de aperfeiçoar as condições sonoras. Além disso, será determinado o tempo de reverberação para a frequência de $500 \mathrm{~Hz}$.

Palavras-chave: Desempenho acústico. Condicionamento acústico. Reverberação.

\section{INTRODUÇÃO}

De maneira geral, as decisões e medidas construtivas são fundamentais para assegurar o condicionamento acústico. Sendo este anteriormente previsto em projeto, mais precisamente no arquitetônico. Outro ponto primordial é o de verificar a orientação e a constituição da fachada, tendo como finalidade a de garantir o conforto acústico do ambiente, nesse caso o concerto/recital (BORGES, 2013).

O condicionamento acústico tem como desígnio o de melhorar a qualidade acústica de qualquer espaço. Propiciando, a melhoria da audição e estímulos sonoros ocasionados no interior do espaço, ou até minimizar o ruído em locais que necessitem de silêncio como hospitais, clínicas e entre outros (CARVALHO, 2006). Mas, sobretudo a ação de distribuir o som, com a frequência, intensidade e altura adequadas, e por consequência ajustando a

1 Engenheiro civil diplomado pela Universidade do Vale do Taquari - Univates. Pós-graduando da Pontifícia Universidade Católica do Rio Grande do Sul PUC RS nos cursos de MBA em Gestão, empreendedorismo e marketing e Pós-graduação em finanças, investimento e banking.

2 Engenheira civil diplomado pela Universidade do Vale do Taquari - Univates.

3 Professor da graduação da Univates. Arquiteto e urbanista diplomado pela Universidade do Vale dos Sinos - UNISINOS. Doutorando em ambiente e desenvolvimento PPGAD pela Universidade do Vale do Taquari - Univates. 
reverberação do local, extinguindo os ecos, sombras sonoras e concentração de som (MOSCATI, 2013).

Isolamento sonoro é uma medida que inibe ou assegura a transmissão sonora, ocasionada por um determinado elemento. Sartori (2016) afirma que a transmissão sonora pode ocorrer por sons aéreos ou por percussão, ou seja, pela estrutura. A reverberação segundo a NBR 12179/1992 define esse termo como o tempo necessário para que o som deixe de ser ouvido, quando extinto a fonte sonora, o som deve sofrer um decréscimo na intensidade de $60 \mathrm{~dB}$.

$\mathrm{O}$ projeto acústico deve ser projeto juntamente com o projeto arquitetônico, pois forma uma única obra. Portanto, o presente estudo apresenta procedimentos para o tratamento acústico de uma sala de concerto e recitais, visando a audibilidade ideal para o ambiente e que atenda a todas as normas vigentes que regularizam este tipo de projeto (ZEIN, 2001).

\subsection{Apresentação do projeto inicial}

O concerto/recital tem capacidade de 90 acentos, do qual é constituído de madeira maciça revestida de tecido camurça e tamanho de 0,45 x 0,45 cm. O espaço possui as seguintes dimensões internas $17,00 \times 10,00 \mathrm{~m}$, o pé direito no palco/tablado (madeira) é de $3,0 \mathrm{~m}$, já na plateia o pé direito é de $3,50 \mathrm{~m}$ e o piso revestido de cerâmica. O teto é de concreto rebocado e pintado de cor escura. A fachada do concerto/recital é composta por janelas convencionais de 10,00 x 3,00 m com vidro liso de 6 mm, já as outras paredes são de alvenaria e revestidas de reboco liso. Figura a seguir representa a planta baixa da sala de concertos/recitais. 
Figura 01 - Planta baixa da edificação.

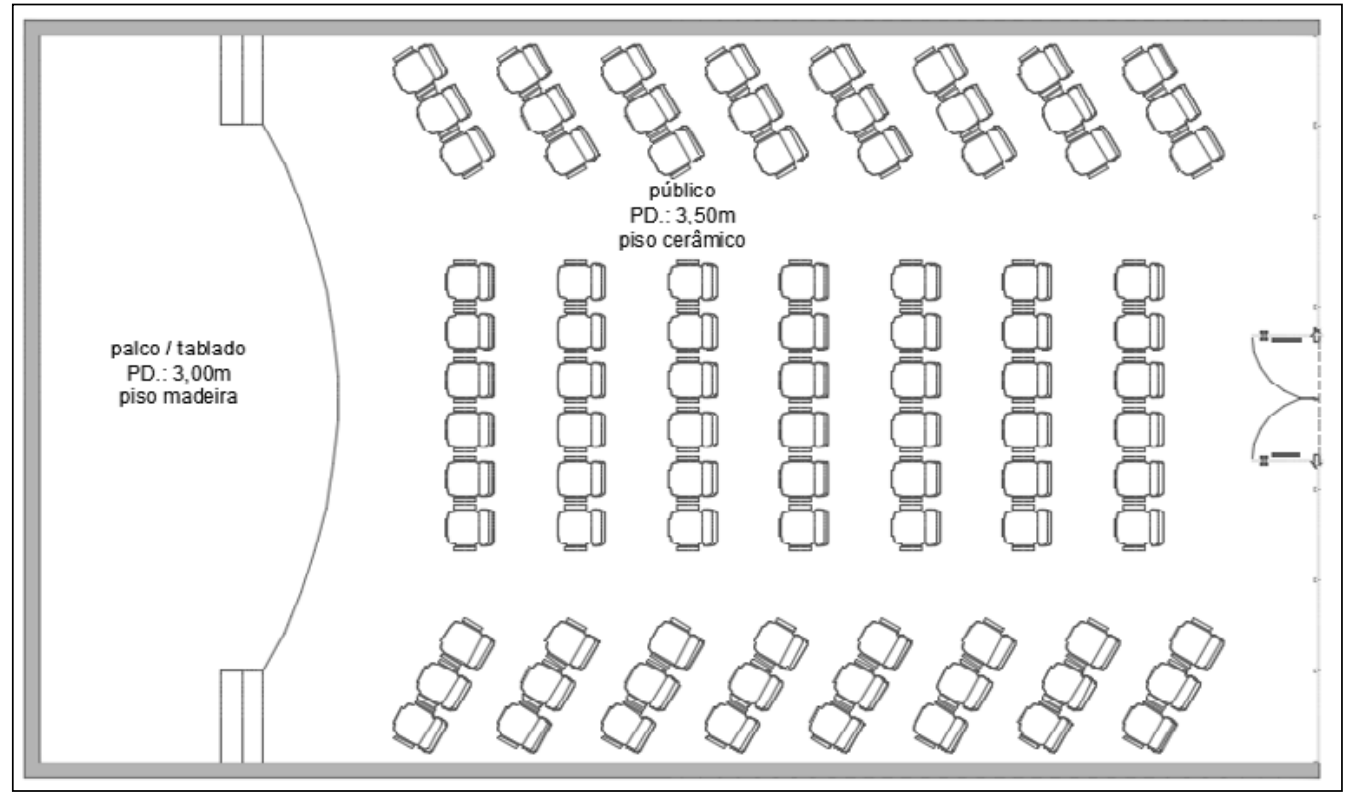

Fonte: Spinelli (2019).

Figura 02 - Corte longitudinal da edificação

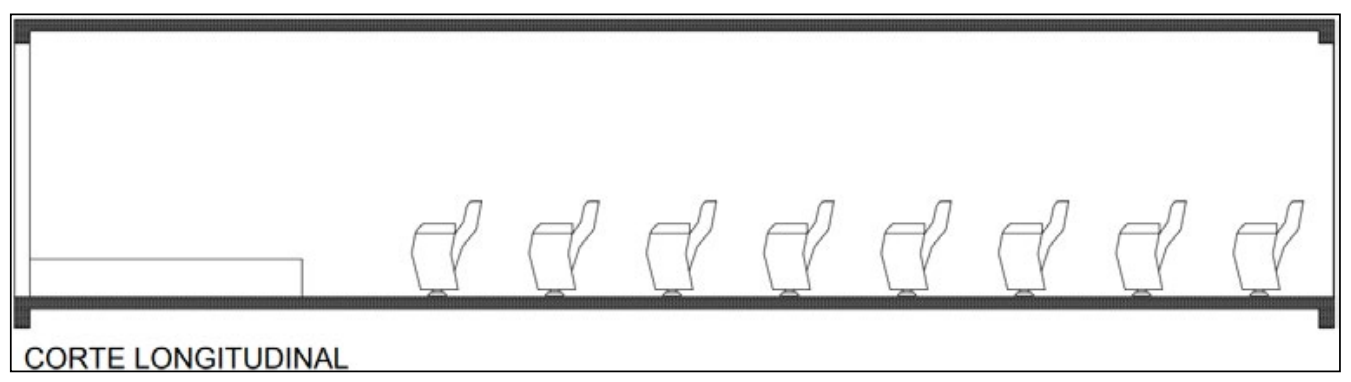

Fonte: Autores(2019)

\section{MEMORIAL DE CÁLCULO DO PROJETO INICIAL}

\subsection{Cálculo do Volume do Ambiente}

Primeiramente calculamos o volume total do espaço.

\subsection{Materiais existentes na sala de concertos/recitais}

Os materiais presentes na sala estão descritos na tabela abaixo. 
Quadro 1- Levantamento dos materiais existentes

\begin{tabular}{|l|}
\hline Materiais Empregados \\
\hline Piso cerâmico \\
\hline Palco de madeira \\
\hline Teto em concreto rebocado e pintado \\
\hline Paredes em alvenaria com reboco liso \\
\hline Janelas convencionais com vidro liso $6 \mathrm{~mm}$ \\
\hline Cadeiras de madeira maciça vazia \\
\hline Vão aberto \\
\hline Cadeiras de madeira maciça ocupada \\
\hline
\end{tabular}

Fonte: Autores (2019).

\subsubsection{Cálculo das áreas superficiais de cada material}

Quadro 2- Áreas superficiais dos materiais

\begin{tabular}{|l|c|}
\hline Materiais Empregados & Superfície $\left(\mathrm{m}^{2}\right)$ \\
\hline Piso cerâmico & 134,93 \\
\hline Palco de madeira & 35,07 \\
\hline Teto em concreto rebocado e pintado & 170,00 \\
\hline Paredes em alvenaria com reboco liso & 154,00 \\
\hline Janelas convencionais com vidro liso 6mm & 26,01 \\
\hline Cadeiras de madeira maciça vazia & 7,29 \\
\hline Vão aberto & 3,99 \\
\hline Cadeiras de madeira maciça ocupada & 10,935 \\
\hline
\end{tabular}

Fonte: Autores (2019).

\subsubsection{Coeficiente de absorção alfa sabine $(\alpha)$}

Os coeficientes de absorção alfa sabine foram retirados na Tabela 1 da NBR 12179/1992, através da frequência de $500 \mathrm{~Hz}$. 
Quadro 3- Coeficientes de absorção das frequências

\begin{tabular}{|l|c|}
\hline Materiais Empregados & $\begin{array}{c}\text { Coeficientes de Absorção p/ a } \\
\text { Frequência 500 Hz }\end{array}$ \\
\hline Piso cerâmico & 0,05 \\
\hline Palco de madeira & 0,25 \\
\hline Teto em concreto rebocado e pintado & 0,02 \\
\hline Paredes em alvenaria com reboco liso & 0,02 \\
\hline Janelas convencionais com vidro liso 6mm & 0,18 \\
\hline Cadeiras de madeira maciça vazia & 0,17 \\
\hline Vão aberto & 1,00 \\
\hline Cadeiras de madeira maciça ocupada & 0,44 \\
\hline
\end{tabular}

Fonte: Autores (2019).

\subsubsection{Absorção sonora}

Conforme a fórmula abaixo, o somatório dos produtos é calculado pela multiplicação da área dos materiais pelos seus respectivos coeficientes de absorção sonora nas frequências indicadas.

Quadro 4- Somatório dos produtos.

\begin{tabular}{|c|c|}
\hline \multicolumn{2}{|c|}{ Absorção Sonora } \\
\hline Materiais & $500 \mathrm{~Hz}$ \\
\hline Piso cerâmico & 6,75 \\
\hline Palco de madeira & 8,77 \\
\hline Cadeiras de madeira maciça ocupada & 4,81 \\
\hline Teto em concreto rebocado e pintado & 3,40 \\
\hline Paredes em alvenaria com reboco liso & 3,08 \\
\hline Janelas convencionais com vidro liso $6 \mathrm{~mm}$ & 4,68 \\
\hline Cadeiras de madeira maciça vazia & 1,24 \\
\hline Vão aberto & 3,99 \\
\hline Total: & 36,72 \\
\hline
\end{tabular}

Fonte: Autores (2019). 


\subsubsection{Tempo de Reverberação atual do ambiente na mesma frequência}

O TR é calculado a partir da fórmula abaixo que leva em consideração o volume e a absorção total do ambiente.

Quadro 5- Tempo de Reverberação atual.

\begin{tabular}{|c|c|}
\hline \multicolumn{2}{|c|}{ Tempo de Reverberação TR } \\
\hline $500 \mathrm{~Hz}$ & 2,61 \\
\hline
\end{tabular}

Fonte: Autores (2019).

\subsubsection{Tempo de Reverberação ideal para o ambiente de $500 \mathrm{~Hz}$}

Para o Tempo de Reverberação a $500 \mathrm{~Hz}$, foi utilizado o gráfico da NBR $12179 / 2000$, sendo considerado a finalidade e o volume do ambiente.

Quadro 6- Tempo de Reverberação ideal para 500 Hz.

\begin{tabular}{|c|}
\hline Tempo de Reverberação Ideal TR $500 \mathrm{~Hz}$ - Sala de Concertos \\
\hline 1,1 \\
\hline
\end{tabular}

Fonte: Autores (2019).

Desta forma é necessário adicionar em média 50,37 sabines à absorção total para a frequência de $500 \mathrm{~Hz}$.

\subsubsection{Zona ideal}

Quadro 7- Zona Ideal.

\begin{tabular}{|c|c|c|}
\hline \multicolumn{3}{|c|}{ Zona Ideal } \\
\hline Frequência & $10 \%$ a menos & $10 \%$ a mais \\
\hline $500 \mathrm{~Hz}$ & 0,99 & 1,21 \\
\hline
\end{tabular}

Fonte: Autores (2019). 


\subsubsection{Comparativo entre o Tempo de Reverberação atual e Tempo de}

Reverberação ideal das frequências.

Quadro 8- Comparativo entre TR atual e TR ideal

\begin{tabular}{|c|c|c|}
\hline \multicolumn{3}{|c|}{ Comparativo de TR } \\
\hline Frequência & TR (s) atual & TR (s) ideal \\
\hline $500 \mathrm{~Hz}$ & 2,61 & 1,1 \\
\hline
\end{tabular}

Fonte: Autores (2019).

\section{MEMORIAL DE CÁLCULO DO PROJETO PROPOSTO}

\subsection{Cálculo do Volume do Ambiente}

Cálculo do volume total do espaço.

\subsection{Materiais propostos para a sala de concertos/recitais}

Os materiais propostos para a sala estão descritos na tabela abaixo.

Quadro 9- Levantamento dos materiais propostos.

\begin{tabular}{|l|}
\hline \multicolumn{1}{|c|}{ Materiais Empregados } \\
\hline Tapete bali macio \\
\hline Palco de madeira \\
\hline Teto de gesso \\
\hline Paredes em alvenaria com chapa de acústica macia diretamente na parede \\
\hline Janelas convencionais com vidro liso $6 \mathrm{~mm}$ \\
\hline Cadeiras de madeira maciça vazia \\
\hline Vão aberto \\
\hline Cadeiras de madeira maciça ocupada (camurça) \\
\hline
\end{tabular}

Fonte: Autores (2019). 


\subsection{Cálculo das áreas superficiais de cada material}

Quadro 10- Áreas superficiais dos materiais.

\begin{tabular}{|l|c|}
\hline \multicolumn{1}{|c|}{ Materiais Empregados } & Superfície $\left(\mathrm{m}^{2}\right)$ \\
\hline Tapete bali macio & 134,93 \\
\hline Palco de madeira & 35,07 \\
\hline Teto de gesso & 170,00 \\
\hline $\begin{array}{l}\text { Paredes em alvenaria com chapa de acústica macia } \\
\text { diretamente na parede }\end{array}$ & 154,00 \\
\hline Janelas convencionais com vidro liso 6mm & 26,01 \\
\hline Cadeiras de madeira maciça vazia & 7,29 \\
\hline Vão aberto & 3,99 \\
\hline Cadeiras de madeira maciça ocupada (camurça) & 10,935 \\
\hline
\end{tabular}

Fonte: Autores (2019).

\subsection{Coeficiente de absorção alfa sabine $(\alpha)$}

Os dados utilizados nos coeficientes de absorção alfa sabine foram retirados na Tabela 1 da NBR 12179/1992, através da frequência 500 Hz.

Quadro 11- Coeficientes de absorção das frequências.

\begin{tabular}{|l|c|}
\hline \multicolumn{1}{|c|}{ Materiais Empregados } & $\begin{array}{c}\text { Coeficientes de Absorção p/ a } \\
\text { Frequência } 500 \mathrm{~Hz}\end{array}$ \\
\hline Tapete bali macio & 0,20 \\
\hline Palco de madeira & 0,25 \\
\hline Teto de gesso & 0,03 \\
\hline $\begin{array}{l}\text { Paredes em alvenaria com chapa de acústica } \\
\text { macia diretamente na parede }\end{array}$ & 0,27 \\
\hline Janelas convencionais com vidro liso 6mm & 0,18 \\
\hline Cadeiras de madeira maciça vazia & 0,17 \\
\hline Vão aberto & 1,00 \\
\hline Cadeiras de madeira maciça ocupada & 0,44 \\
\hline
\end{tabular}

Fonte: Autores (2019). 


\subsection{Somatório dos produtos das diversas áreas pelos seus respectivos coeficientes de absorção sonora}

Conforme a fórmula abaixo, o somatório dos produtos é calculado pela multiplicação da área dos materiais pelos seus respectivos coeficientes de absorção sonora nas frequências indicadas.

Quadro 12- Somatório dos produtos

\begin{tabular}{|l|c|}
\hline \multicolumn{2}{|c|}{ Absorção Sonora } \\
\hline \multicolumn{1}{|c|}{ Materiais } & $500 \mathrm{~Hz}$ \\
\hline Tapete bali macio & 26,99 \\
\hline Palco de madeira & 8,77 \\
\hline Cadeiras de madeira maciça vazia & 1,24 \\
\hline Teto de gesso & 5,10 \\
\hline $\begin{array}{l}\text { Paredes em alvenaria com chapa de acústica macia diretamente } \\
\text { na parede }\end{array}$ & 41,58 \\
\hline Janelas convencionais com vidro liso 6mm & 4,68 \\
\hline Cadeiras de madeira maciça ocupada & 4,81 \\
\hline Vão aberto & 3,99 \\
\hline Total: & 97,16 \\
\hline
\end{tabular}

Fonte: Autores (2019).

\subsection{Tabela com o Tempo de Reverberação atual do ambiente na mesma frequência}

O TR é calculado a partir da fórmula abaixo que leva em consideração o volume e a absorção total do ambiente.

Quadro 13- Tempo de Reverberação atual

\begin{tabular}{|c|c|}
\hline \multicolumn{2}{|c|}{ Tempo de Reverberação TR } \\
\hline $500 \mathrm{~Hz}$ & 0,99 \\
\hline
\end{tabular}

Fonte: Autores (2019).

\subsection{Tempo de Reverberação ideal para o ambiente de $500 \mathrm{~Hz}$}

Para o Tempo de Reverberação a $500 \mathrm{~Hz}$, foi utilizado o gráfico da NBR $12179 / 2000$, sendo considerado a finalidade e o volume do ambiente. 
Quadro 14- Tempo de Reverberação ideal para $500 \mathrm{~Hz}$

\begin{tabular}{|c|}
\hline Tempo de Reverberação Ideal TR $500 \mathrm{~Hz}$ - Sala de Concerto \\
\hline 1,1 \\
\hline
\end{tabular}

Fonte: Autores (2019).

Desta forma o valor obtido na faixa de $500 \mathrm{~Hz}$ foi de 97,16 sabines, valor este superior ao de 87,09 sabines necessários.

\subsection{Zona ideal}

Quadro 15- Zona Ideal

\begin{tabular}{|c|c|c|}
\hline \multicolumn{3}{|c|}{ Zona Ideal } \\
\hline Frequência & $10 \%$ a menos & $10 \%$ a mais \\
\hline $500 \mathrm{~Hz}$ & 0,99 & 1,21 \\
\hline
\end{tabular}

Fonte: Autores (2019).

\subsection{Comparativo entre o Tempo de Reverberação atual e Tempo de} Reverberação ideal das frequências.

Quadro 16- Comparativo entre TR atual e TR ideal

\begin{tabular}{|c|c|c|}
\hline \multicolumn{3}{|c|}{ Comparativo de TR } \\
\hline Frequência & TR atual & TR ideal \\
\hline $500 \mathrm{~Hz}$ & 0,99 & 1,1 \\
\hline
\end{tabular}

Fonte: Autores (2019).

3.10 Comparativo entre o Tempo de Reverberação do projeto inicial e o Tempo de Reverberação do projeto proposto das frequências.

Quadro 17- Comparativo entre TR inicial e TR melhoria

\begin{tabular}{|c|c|c|}
\hline \multicolumn{3}{|c|}{ Comparativo de TR } \\
\hline Frequência & TR inicial & TR melhorias \\
\hline $500 \mathrm{~Hz}$ & 2,61 & 0,99 \\
\hline
\end{tabular}

Fonte: Autores (2019). 


\section{CONCLUSÃO}

Conclui-se através dos resultados obtidos inicialmente, que o projeto não atendeu a NBR 12179/1995 que regulariza as salas de concerto/recital, no parâmetro de reverberação, além disso, o resultado encontrado excedeu a zona ideal de condicionamento acústico.

Posteriormente, as adaptações dos materiais empregados nos revestimentos (teto, forro, parede e piso) e a troca das cadeiras. Por consequência se constatou uma melhoria significativa na acústica do concerto/recital. Alcançando os parâmetros estipulados pela norma, ou seja, se enquadrando na zona ideal.

\section{REFERÊNCIAS}

ASSOCIAÇÃO BRASILEIRA DE NORMAS TÉCNICAS. NBR 12179 - Tratamento acústico em recintos fechados. Rio de Janeiro: ABNT, 1992.

BORGES, R. M. Análise de desempenho térmico e acústico de unidades habitacionais construídas no conjunto habitacional Benjamin José Cardoso em Viçosa-MG. Minas Gerais, 2013. Dissertação (Magister Scientiae em Engenharia Civil).

CARVALHO, Régio Paniago. Acústica arquitetônica. Brasília: Thesaurus, 2006.

MOSCATI, S. R. Desempenho acústico de templos e igrejas: subsídios à normalização. São Paulo, 2013. Dissertação (Mestre em Arquitetura e Urbanismo).

SARTORI, L. Isolamento e condicionamento acústico de um salão de festas em um prédio residencial. Porto Alegre, 2016. Tese (Engenharia Civil).

ZEIN, R. V. Sala São Paulo de Concertos. São Paulo: Alter Market, 2001. 